Friebe

\title{
Endoscopic filter fluorometer for emission detection of Protoporphyrin IX and its direct precursors in PDT and PDD
}

\begin{abstract}
Photodynamic therapy (PDT) is a potential option for treatment of cancer since it can be performed non- invasive for superficial cancers or minimal-invasive with low traumatization. But PDT is intrinsically inefficient due to the complex accumulation of the photosensitizing drug inside the tumor and the processes of heme syntheses to create the needed cell killing components. To optimize the outcome of PDT and increase acceptance as viable option it is necessary to predict the optimal time for the start of the treatment based on measurable precursors. A former cell study proposed a new filter fluorometer in a complex and sensitive setup. In this work we now designed and tested a simplified system that can be used in combination with standard endoscopic imaging systems. This system will be used as base to prove viability of this approach for a future clinical study.
\end{abstract}

Keywords: photodynamic therapy, endoscopic systems, PPIX, CP-III, urology, bladder cancer

https://doi.org/10.1515/cdbme-2020-3150

\section{Introduction}

In 2016, the German Cancer Registry (ZfKD) estimates that around 492,000 cancers were diagnosed in Germany [1]. For the variety of cancer types several treatment options are available. Surgical tumor resection is still the most important first treatment strategy. One of the treatment alternatives, when surgery is not applicable, is Photodynamic Therapy

\footnotetext{
${ }^{*}$ Corresponding author: Axel Boese: Otto-von-Guericke University Magdeburg, Medical Faculty, Leipziger Str. 44, Magdeburg, Germany, e-mail: axel.boese@ovgu.de Alexander Wagner, Michael Friebe: Otto-von-Guericke University Magdeburg, Medical Faculty, Magdeburg, Germany Uwe Bernd Liehr, Jakob Johann Wendler: University Clinic Magdeburg, Clinic for Urology, Magdeburg, Germany
}

(PDT). PDT is approved for treatment of skin, lung or oesophageal cancer but is also tested in bladder cancer [2]. PDT has an increased relapse rate however due to the complex metabolism in the cells. PDT starts with the admission of a photosensitizing drug, such as 5-aminolevulinic acid (5-ALA) or hexaminolevulinate hydrochloride (HAL, a derivative of 5ALA) that accumulates over time in the tumor tissue and metabolizes to protoporphyrin IX (PP-IX). When the tissue is exposed to light, an active form of oxygen (radical) that destroys nearby cancer cells is produced [3]. For a high efficacy of the treatment the ratio of PP-XI, light and oxygen has to be optimal at the time of light exposure. The presence of PP-IX can be evaluated by fluorescence imaging. PP-IX emits a strong red fluorescence at a peak of 630-635 nm when excited by a $405 \mathrm{~nm}$ blue light.

The metabolic process creates two more porphyrins as precursors, Coproporphyrin III (CP-III) and Uroporphyrin III (UP-III). Both substances have a fluorescence peak at approx. $10 \mathrm{~nm}$ below the peak of PP-IX. Since these precursors appear timingwise before the highest presence of PP-IX they can be used as predictor for the optimal time slot for the cell killing light exposure. This could lead to a higher efficacy of PDT.

In prior work on cell models we could show that these precursors are detectable using special optical filters during fluorescence imaging using a custom-build, but rather complex and sensitive filter fluorometer $[4,5]$. For use in a future clinical setup a less complex and less sensitive system has to be build that integrates the technology into available standard medical equipment used within the clinical workflow.

Some endoscopic systems already allow fluorescence imaging for photodynamic diagnostics (PDD). PDD uses similar drugs (Hexvix, Ipsen Pharma, France) and heme metabolism like PDT to create fluorescence of tumor cells. Thus, a fast detection of cancerous areas and treatment control in endoscopic imaging can be supported. PDD is a standard procedure in urology to diagnose bladder cancer [6]. We want to use this standard procedure to prove our hypothesis of the detectability of the precursors CP-III and UP-III by filtered 
fluorescence imaging. In this work we describe a new filter setup that can be integrated into endoscopic imaging systems considering the requirements for a clinical applicable device.

\section{Materials}

\subsection{Observation and requirements analysis}

For design of a system that meets the need of the clinical application, a user integrated approach based on [7] was performed. Today PDD is used in urology to detect forms of bladder cancer [6]. To develop an efficient concept, we observed urologists using a PDD system (SCB Image 1, Karl Storz, Germany) for transurethral bladder resection (TURB). For PDD at the bladder, Hexvix (Ipsen Pharma, France) is applied inside the bladder one hour before the diagnostic procedure [8]. This time is needed for accumulation in the tissue and the metabolic process to produce PP-IX. A resectoscope including endoscopic imaging is inserted into the bladder. First normal white light inspection is performed. In a second step the system is switched into PDD mode using $405 \mathrm{~nm}$ blue light. In our observation fluorescence was rare even though a clear finding was made in normal imaging. To prove our hypothesis, we have to consider this uncertainty.

Based on the observation and discussion with experienced users the following basic conditions for the design were defined:

$\square \quad$ Regular diagnostic should not be influenced

$\square \quad$ Placement of a filter system between endoscope and camera as preferred place

$\square \quad$ Placement under sterile cover is a limiting factor for the size of the new system

$\square \quad$ Use of standard endoscopic connectors

$\square \quad$ Stable one hand use should be possible under sterile cover

\subsection{General concept}

To allow standard diagnostics and the parallel acquisition of filtered images we propose the following workflow:

A new designed filter system will be placed between the sterile endoscope and the non-sterile camera under the separating sterile cover. The filter system allows an easy change from filtered view to standard view without disassembly. Two filters have to be integrated, a $630 \mathrm{~nm}$ filter for PP-IX separation and a $620 \mathrm{~nm}$ for CP-III and UP-III detection.
After application of Hexvix the endoscope is directly placed inside the bladder and images are acquired every 5 minutes with both filters and standard view for one hour total allowing the observation of accumulation and metabolism over time.

\subsection{Prototype}

Based on the criteria already described above, a prototype filter box was designed. This box includes a slider that contains 3 sections. 2 sections are intended for the filter glasses. The middle section provides an open channel to allow standard imaging. The filter box was equipped with standard eyepiece connectors for endoscopes according to [9] to connect endoscope on one side and camera on the other. We created the filter prototype in Solidworks ${ }^{\circledR}$ and built it using a 3D printer (Prusa ${ }^{\circledR}$ FDM Printer, Czech Republic). Two optical bandpass filters are mounted into the slider, FB620-10 and FB630-10 (Thorlabs, Germany). The filter box prototype can be seen in Figure 1.

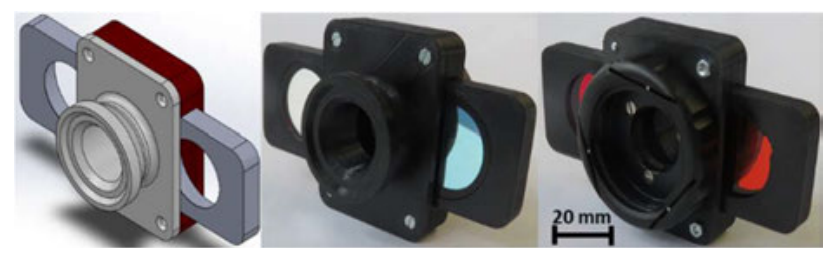

Figure 1: Design and realization of the filter box

The filter box is made of biocompatible PET with dimensions of $55 \times 94 \times 13 \mathrm{~mm}$. PET is resistant to ethanol and acetone allowing disinfection. To separate the camera and filter box from the sterile endoscope, the use of a standard sterile cover including eyepiece connectors (Quick Change Camera Drape, P3 Medical Ltd, UK) is mandatory. The complete prototype setup including endoscopic imaging is shown in Figure 2.

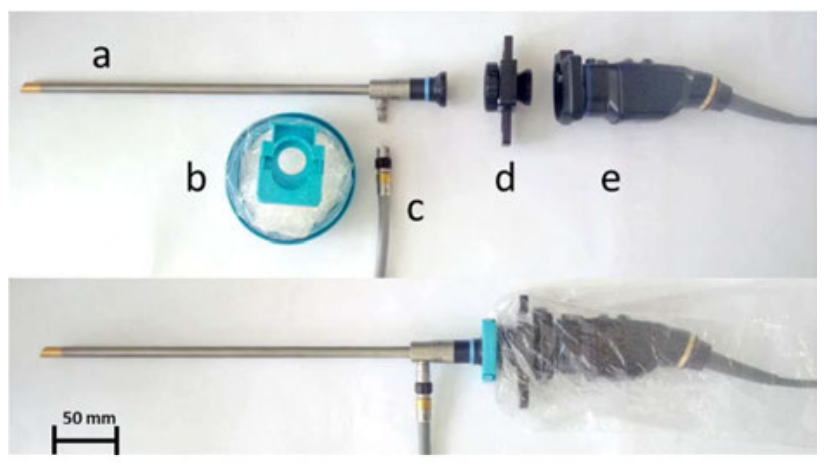

Figure 2: Prototype setup including a) endoscope, b) sterile cover, c) light connector, d) filter box, e) camera 


\subsection{Testing}

In order to use the filter box later successfully in a clinical study, we tested basic functionality on PP-IX and CP-III samples under laboratory conditions. Therefore, the imaging and filter setup were placed inside a lockable, dark box. A laser (PPM110 (405-125), Power Technology, USA) provided a $405 \mathrm{~nm}$ light beam for excitation. A standard endoscopy system (EVIS EXERA III, Olympus, Germany) was used for image acquisition. Samples of liquid PP-IX and CP-III [5] were placed aligned to the laser beam. The test setup is shown in Figure 3.

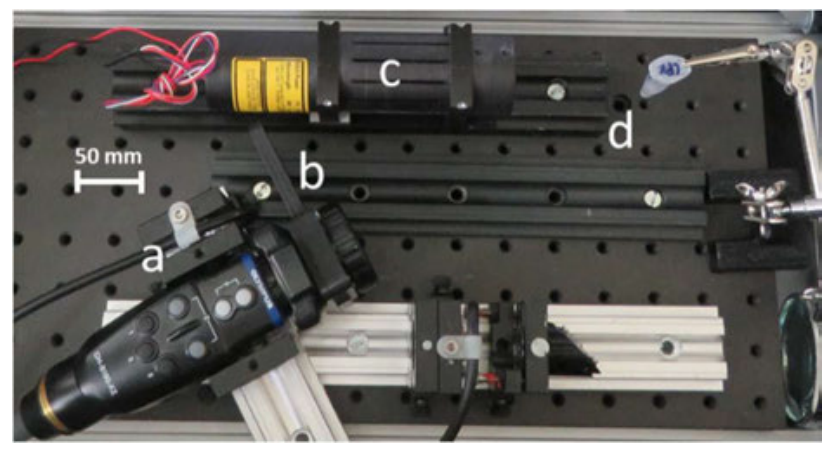

Figure 3: The test setup: a) camera, b) filter box, c) laser, d) test sample

First, the samples were irradiated with the laser and imaged without additional filters. Thus, the laser beam position was aligned. The emitted light is visible as fluorescence as shown in Figure 4.

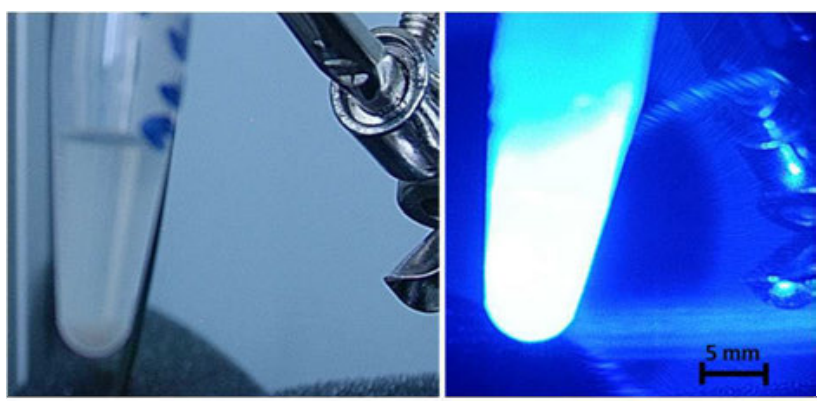

Figure 4: Detailed view of the test samples with and without fluorescence

After the alignment procedure, the PP-IX sample was imaged with the $620 \mathrm{~nm}$ bandpass filter first and with the $630 \mathrm{~nm}$ bandpass filter afterwards. The same procedure was repeated for the CP-III sample. All acquired images were analysed using IMAGEJ. A simple line profile plot was used to visualize any differences in fluorescence intensity. All fluorescence images were obtained under identical conditions. Additionally to the functional testing's, we evaluated general usability and safety of the system.

\section{Results and discussion}

A new filter box to distinguish different fluorescence wavelength of porphyrins was developed. It can be used with standard endoscopic imaging equipment. It fulfills the basic requirements defined before. The connectors between camera, filter, sterile cover and endoscope are stable and the connection is reliable. The assembly of the filter box in combination with the sterile cover causes no additional effort. The use of the slider of the filter box under the cover is possible even with one hand.

The imaging tests with the filter box demonstrated the expected functionality. According to the literature the fluorescence of PP-IX and CP-III differ in wavelengths. PPIX emits a fluorescence peak at $630 \mathrm{~nm}$ and the CP-III at approximately $620 \mathrm{~nm}$. We imaged both samples using both filters. It was expected that PP-IX shows higher fluorescence in combination with $630 \mathrm{~nm}$ filter and lower with $620 \mathrm{~nm}$ filter and vice versa for CP-III. This was proven with our experiments as shown in Figure 5 and 6 and summarized in Table 1.

Table 1: Comparison of fluorescence of PP-IX and CP-III using $620 \mathrm{~nm}$ and $630 \mathrm{~nm}$ bandpass filters

\begin{tabular}{lll}
\hline Filter & PP-IX intensity & CP-III intensity \\
\hline $620 \mathrm{~nm}$ & 80 & 125 \\
$630 \mathrm{~nm}$ & 100 & 90 \\
\hline
\end{tabular}
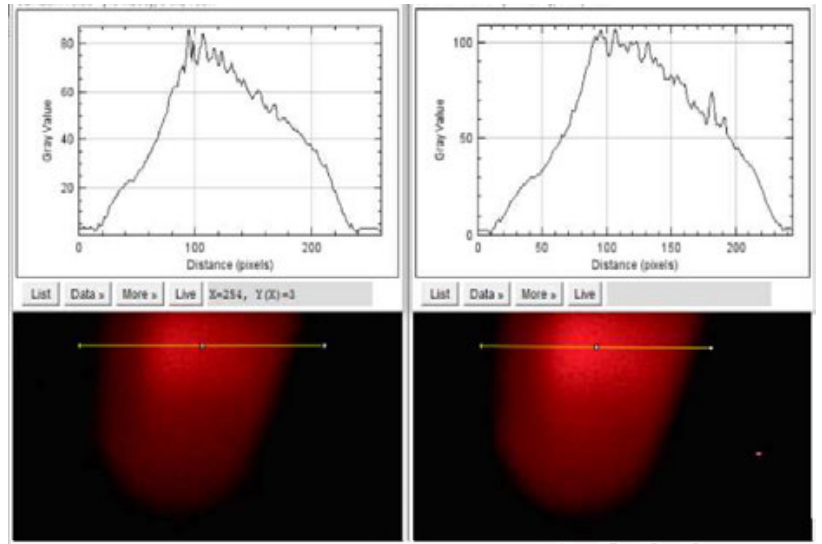

Figure 5: PP-IX measurement with filter 620nm (left) and $630 \mathrm{~nm}$ (right) 


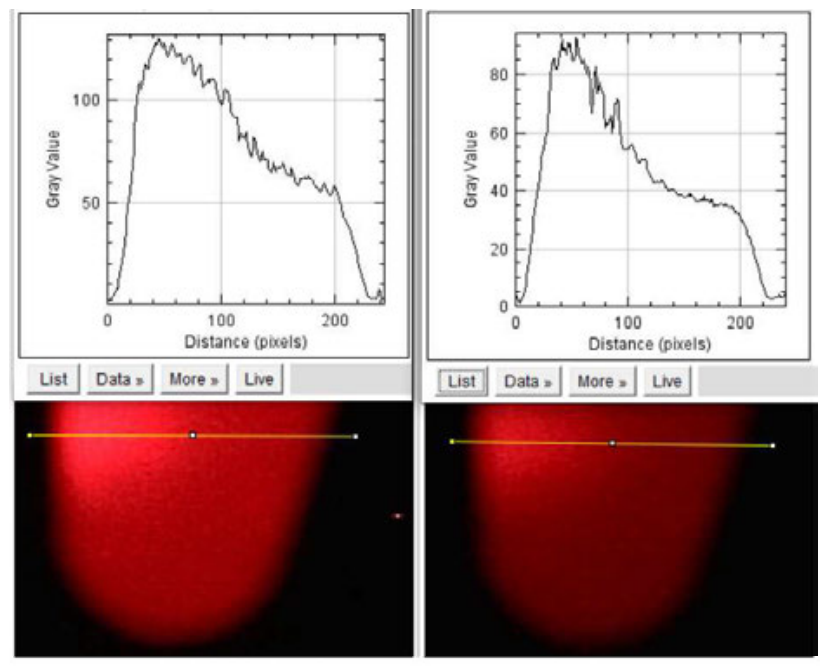

Figure 6: CP-III measurement with filter 620nm (left) and 630nm (right)

\section{Conclusion}

A new filter system for endoscopic systems to differentiate PPIX and CP-III fluorescence was built and tested. We could confirm the general functionality on first test samples. The design based on clinical observation lead to a system that can be used in a clinical application. In a next step we will test the system in combination with a standard PDD system and - after ethical approval - in a clinical study on bladder cancer. These tests will help to prove our hypothesis that it is possible to measure and predict the optimal time slot for the cell killing light application in PDT.

\section{Author Statement}

Research funding: The author state no funding involved. Conflict of interest: Authors state no conflict of interest. Informed consent: Informed consent has been obtained from all individuals included in this study. Ethical approval: The research related to human use complies with all the relevant national regulations, institutional policies and was performed in accordance with the tenets of the Helsinki Declaration, and has been approved by the authors' institutional review board or equivalent committee.

\section{References}

[1] R. Koch-Institut und G. der epidemiologischen K. in D. e.V, „Krebs in Deutschland 2015/2016“, 2019, doi: http://dx.doi.org/10.25646/5977.

[2] N. Yavari, S. Andersson-Engels, U. Segersten, und P.-U. Malmstrom, „An overview on preclinical and clinical experiences with photodynamic therapy for bladder cancer", Can J Urol, Bd. 18, Nr. 4, S. 5778-5786, Aug. 2011.

[3] J. Dobson, G. F. de Queiroz, und J. P. Golding, „Photodynamic therapy and diagnosis: Principles and comparative aspects“, Vet. J., Bd. 233, S. 8-18, 2018, doi: $10.1016 /$ j.tvj1.2017.11.012.

[4] R. Landes, A. Illanes, D. Goeppner, H. Gollnick, und M. Friebe, „A study of concentration changes of Protoporphyrin IX and Coproporphyrin III in mixed samples mimicking conditions inside cancer cells for Photodynamic Therapy“, PLOS ONE, Bd. 13, Nr. 8, Aug. 2018, doi: 10.1371/journal.pone.0202349.

[5] R. Landes, A. Illanes, A. van Oepen, D. Goeppner, H. Gollnick, und M. Friebe, ,Fiber-optic filter fluorometer for emission detection of Protoporphyrin IX and its direct precursors - A preliminary study for improved Photodynamic Therapy applications", Results in Physics, Bd. 8, S. 1232-1233, März 2018, doi: 10.1016/j.rinp.2018.01.059.

[6] Z. Tandogdu $u$. a., „Photodynamic versus white lightguided treatment of non-muscle invasive bladder cancer: a study protocol for a randomised trial of clinical and cost-effectiveness“, BMJ Open, Bd. 9, Nr. 9, S. e022268, Sep. 2019, doi: 10.1136/bmjopen-2018-022268.

[7] A. Boese, Lösungsfindung mit dem Endnutzer, ein neuer Ansatz in der methodischen Produktentwicklung am Beispiel der Medizintechnik. Aachen, Germany: Shaker, 2016., ISBN 978-3-8440-4947-3

[8] Karl Storz, „Karl Storz Bluelight Cystoscopy with Cystview".

[9] ISO/TS 18339:2015-11 - Endotherapy devices - Eyepiece cap and light guide connector. 